\title{
Mesonic Potential Theory for High Temperature Superconductivity
}

\section{Author}

Tanay Shardul Gangan

\section{Addresses}

Tanay Shardul Gangan, Email - tanay.gangan@gmail.com Institute - I.E.S Orion, Dadar (E), Mumbai - 400014, Maharashtra. Residence - A / 403, Jigna Apartment, N.M. Joshi Marg, Currey Road (W), Mumbai - 400013, Maharashtra.

\section{Acknowledgements}

I would sincerely like to thank Dr. Shardul Sham Gangan and Miss Nandita Shardul Gangan for their moral support. Then I would like to express my gratefulness to Master Avdhoot Ashish Lendhe and Dr. Shreya Sushil Shetye for their helpful reviews on this paper.

\section{Abstract}

Present study proposes a mesonic potential theory as an attempt to provide a quantum theory of superconductivity. The results obtained will be a well description of a potential well of the particle concerned, i.e. the meson, and predictions based on the theory regarding which elements can be used as high temperature superconductors for technological advancements. The results will be obtained by carrying of a thorough calculation and understanding of the pre-defined quantum postulates involved in potential, including quantities like Bound State Energies, Energy Shift, Nuclear Structure Compilation, etc. The study will be using various pre - defined formulae for the calculation of quantities and will be relating them to the theory which is being proposed.

\section{Keywords}

Mesons; High Temperature Superconductivity; YBaCuO; Potential well; Nuclear Shell Theory

\section{Declarations}

Funding: No funding received

Conflicts of Interest: No conflicts

Availability of data and material: Not applicable

Code Availability: Not applicable

Authors' contributions: only one author involved 


\section{Contents}

Theory in Brief and necessary formulae.

03

Lattice Structure Analysis and Nuclei Study. 04

Calculation of Mesonic Quantities. 06

Potential Well Understanding. 10

Conclusion.

References. 


\section{Theory in Brief and Necessary formulae}

The BCS theory, that is the current theory of superconductivity does not provide a quantum theory for the explanation to the phenomenon of superconductivity occurring at temperatures higher than absolute zero even though this type of superconductivity has been experimentally found. In the past year there have been attempts to explain the phenomenon of high temperature superconductivity by the conventional approach, i.e., the electron - phonon - nuclear interactions. One such paper is published by a Russian physicist Viktor Lakhno from Keldysh Institute of Applied Mathematics, by taking a bipolaron as a basis. A polaron is a quasiparticle that consists of electrons and phonons and can form pairs due to electron - phonon interactions.

However to conquer the problem of excitation regarding the pairing of cooper pairs, involves a certain particle for the mediation of charge as a bosonic superfluid with zero resistance. Thus, the particle discussed here is the meson. This particle was used to describe the nuclear force which binds the protons and neutrons together in the nucleus of an atom. Therefore, in this paper direct electron - nuclear interactions have been discussed as a possibility to explain high temperature superconductivity. Many, more types and variations have been made with respect to this particle but we will be studying about one particular meson namely the negatively charged pion which has the spin matrix as follows...

$$
\emptyset \text { matrix }=\left(\begin{array}{cc}
0 & 0 \\
1 & -1
\end{array}\right) \quad 1.1
$$

Thus, it can behave in the form of a bosonic superfluid. It has the mass of $139 \mathrm{Mev} \cdot \mathrm{c}^{-2}$. So if at all it has been produced to due electron interactions with the nucleus of an atom the energy produced would cause the critical temperature of the superconducting material to rise considerably.

The most important of all the various formulae we will be using are -

The free Schrödinger equation -

$$
\left(\frac{\hbar^{2} \nabla^{2}}{2 m}+V(r)\right) \psi=E \psi \quad 1.2
$$

Bound State Energy

Where $N_{0}$ is a constant as-

$$
\Delta \sim \hbar \omega \exp \left(\frac{-1}{N_{0} V_{0}}\right) \quad 1.3
$$

$$
N_{0}=\left(\frac{m P_{f}}{4 \pi^{2} \hbar^{3}}\right) \quad 1.4
$$

Ground State Energy 


$$
k \tan k L=\sqrt{\beta^{2}-k^{2}}
$$

Where,

$$
\begin{array}{cc}
E=\frac{\hbar^{2} k^{2}}{2 m} & 1.6 \\
\beta=\sqrt{\frac{2 m U_{0}}{\hbar^{2}}} & 1.7
\end{array}
$$

High Temperature superconductors are superconductors in which the superconductive state occurs at around $100 \mathrm{~K}$ and are not described by the BCS theory of superconductivity. Here we will be examining Yttrium Barium Copper Oxide (YBaCuO)superconductor. Some other examples of High Temperature Superconductors are -

- Mercury Barium Calcium Copper Oxide ( $\mathrm{HgBaCaCuO})$

- Mercury Titanium Barium Calcium Copper Oxide (HgTlBaCaCuO) 


\section{Lattice Structure Analysis and Nuclei Study. 3.1. Lattice of $\mathrm{YBaCuO}$}

This study focuses on one particular high temperature superconductor as an example for further calculations. The lattice of $\mathrm{YBaCuO}$ is given below, (obtained from www.wikipedia.org).

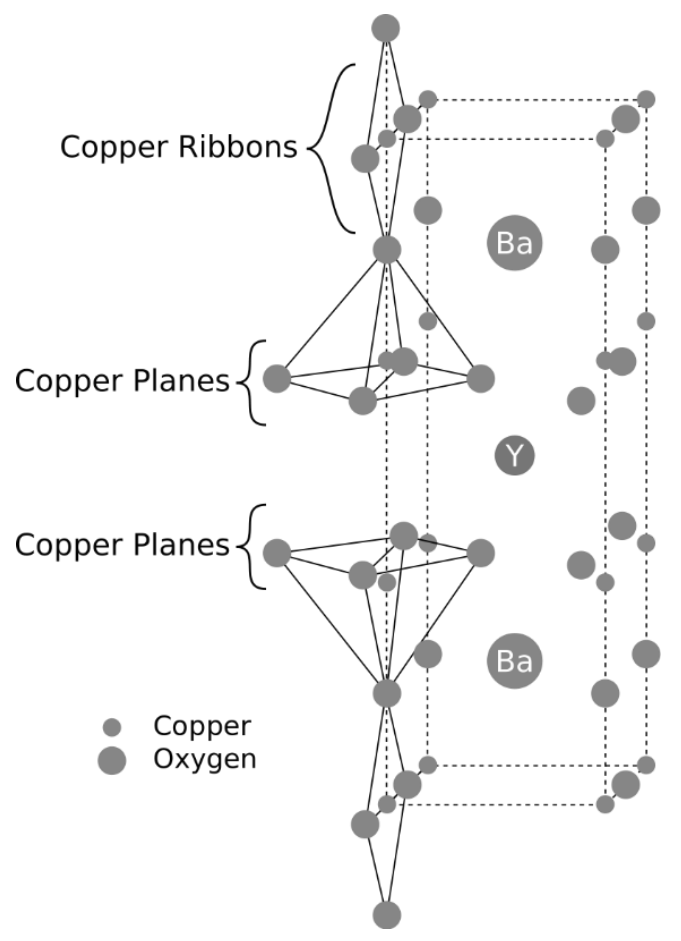

Lattice of $\mathrm{YBaCuO}$

(Reference : www.wikipedia.org, Rswarbrick - Own work, based on Wikipedia's Ybco002.jpg)

Fig. 2.1

From the structure and our particle considerations we can say that either one of the 3 metallic substances with relatively heavy nuclei have chances of producing a meson due to interactions occurring at low temperature. To conclude which nuclei is producing mesons we need to perform a thorough study of each of their nuclei with respect to the nuclear shell theory [3].

First let us consider Barium,

$\mathrm{Z}=39$

We know the nuclear orbits with increasing nucleon numbers, i.e., $\pi_{1}, \pi_{2}, \pi_{3}$, etc [3].

Thus its last nuclear shell will be $\pi_{7}$ with its final orbits having 7 and 6nucleons respectively. Thus, the final orbit has 6 nucleons which are stable in themselves and are very less likely to undergo changes [3].

Similarly if we consider $\mathrm{Cu}$, Its final orbits of final shell $\pi_{5}$ are $5,5 \& 4$ respectively whose final orbit is again stable [3]. 
The next one left is Yttrium which interestingly after similar analysis has only 1 nucleon in its final orbit thus is susceptible for the emission of a meson [3]. Thus, we can say that a flowing electron interacts with Yttrium nuclei in $\mathrm{YBaCuO}$ to produce a meson which at low temperature has the state of a Bose - Einstein Condensate thus carrying the negative charge for balancing the charge without any resistance and the meson further disintegrates to give an electron itself. While the disintegration of the meson a neutrino is released which excites the emitted electron. We can draw a diagram to show this as follows -

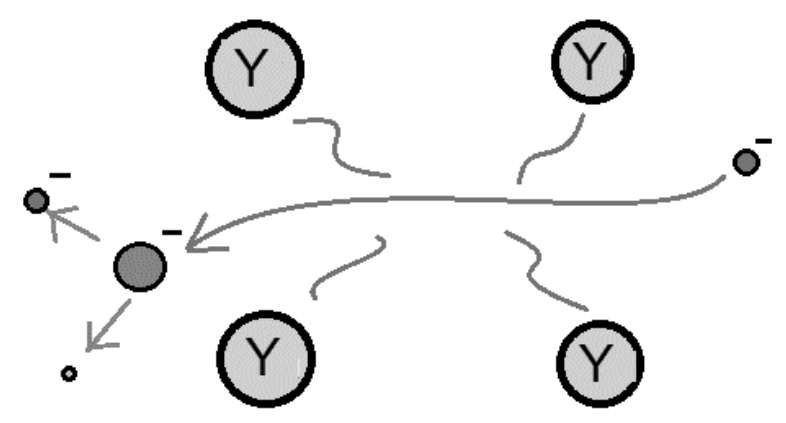

Fig. 2.2, A diagram representing the interaction of an electron with Yttrium atoms to produce a negative pion which in-turn disintegrates to form an electron and a neutrino.

According to this theory, the elements which should be present in high temperature superconductors taking the nuclear shell theory as a base in increasing order of their atomic masses are -

- Hydrogen $(\mathrm{H} ; \mathrm{Z}=1)$

- $\operatorname{Lithium}(\mathrm{Li} ; \mathrm{Z}=7)$

- $\operatorname{Beryllium~}(\mathrm{Be} ; \mathrm{Z}=9)$

- Calcium $(\mathrm{Ca} ; \mathrm{Z}=20)$

- $\operatorname{Vanadium}(\mathrm{V} ; \mathrm{Z}=51)$

- $\operatorname{Iron}(\mathrm{Fe} ; \mathrm{Z}=56)$

- $\operatorname{Yttrium}(Y ; Z=89)$

- Indium (In; $Z=115)$

- $\quad$ Iodine $(\mathrm{I} ; \mathrm{Z}=127)$

- Praseodymium (Pr; $Z=141)$

- $\operatorname{Gadolinium~}(\mathrm{Gd} ; \mathrm{Z}=157)$

If at all this theory is proved correct, it will be much consolation that iron is a part of the list! 


\section{Calculating Mesonic Quantities.}

Before, starting the calculations let us take a look at all the formulae we will be using.,

$$
\begin{gathered}
k_{\pi}=\frac{\hbar^{2} \nabla_{\pi}^{2}}{2 m} \\
\Delta=\hbar \omega \exp \left(\frac{-1}{N_{0} V_{0}}\right)
\end{gathered}
$$

We will not be using the formulae for the kinetic energy and the formula for the potential energy since they can be directly derived from the total energy and experimental evidence respectively. Thus, the total energy of an emitted meson is $549 \mathrm{MeV}$ and its potential energy is $139 \mathrm{MeV}$.

Therefore, its kinetic energy will be,

$$
\begin{gathered}
k=E-V(r) \quad 3.3 \\
k=549-139=410 \mathrm{MeV} \quad 3.4
\end{gathered}
$$

We have for momentum,

$$
\begin{gathered}
p=\sqrt{k 2 m} \quad 3.5 \\
p=\sqrt{410 \times 2 \times 139} \quad 3.6 \\
p=337.6 \mathrm{MeV} \cdot \mathrm{c}^{-1} \quad 3.7 \\
p=1.8 \times 10^{-19} \mathrm{kgms}^{-1} \quad 3.8
\end{gathered}
$$

And we know,

$$
m=139 \mathrm{MeV} \cdot \mathrm{c}^{-2}=2.488 \times 10^{-28}
$$

Let us now breakdown the equation-

$$
\Delta \sim \hbar \omega \exp \left(\frac{-1}{N_{0} V_{0}}\right)
$$

Since,

$$
N_{0}=\frac{p_{f} m}{4 \pi^{2} \hbar^{3}}
$$

Therefore, 


$$
N_{0} V_{0}=\frac{P_{f} m V_{0}}{4 \pi^{2} \hbar^{3}} \quad 3.12
$$

As we know,

$$
\begin{gathered}
V_{0}=139 \mathrm{MeV}=2.224 \times 10^{-11} \mathrm{~J} \\
N_{0} V_{0}=\frac{1.8 \times 10^{-19} \times 2.488 \times 10^{-28} \times 2.224 \times 10^{-11}}{4 \times 3.14^{2} \times 1.05457^{3} \times 10^{-102}} \\
N_{0} V_{0}=\frac{1.8 \times 2.488 \times 2.224}{4 \times 3.14^{2} \times 1.05457^{3}} \times 10^{44} 3.15 \\
N_{0} V_{0}=2.15 \times 10^{43} 3.16
\end{gathered}
$$

Thus,

$$
\frac{-1}{N_{0} V_{0}}=-4.65 \times 10^{-43}
$$

Therefore,

$$
\exp \left(\frac{-1}{N_{0} V_{0}}\right) \approx 1
$$

Hence,

$$
\Delta=\hbar \omega \quad 3.19
$$

And,

$$
\lambda=\frac{2 h c}{2 E} \quad 3.20
$$

We take $2 \mathrm{E}$ as two photons are released on the disintegration of a meson.

$$
\begin{array}{r}
2 E=m_{\pi} c^{2} \\
\lambda=\frac{2 h c}{m_{\pi} c^{2}} \quad 3.21 \\
\lambda=\frac{2 \times 6.6 \times 10^{-34} \times 3 \times 10^{8}}{139}
\end{array}
$$

And, 


$$
\begin{gathered}
\omega(\text { frequency })=\frac{c}{\lambda} \quad 3,24 \\
\omega=\frac{3 \times 10^{8} \times 139}{2 \times 6.6 \times 10^{-34} \times 3 \times 10^{8}} \\
\omega=10.53 \times 10^{34} \quad 3.26
\end{gathered}
$$

Substituting,

$$
\begin{gathered}
\Delta=\hbar \omega \quad 3.27 \\
\Delta=1.05457 \times 10^{-34} \times 10.53 \times 10^{34} \\
\Delta=11.104 \mathrm{MeV} \quad 3.29
\end{gathered}
$$

Therefore, we have the bound state energy of the emitted meson at nearly $11 \mathrm{MeV}$. We also have the total energy of the superconductive state of the meson as-

$$
E=549-11=538 \mathrm{MeV} \quad 3.30
$$

The apparent spin matrix which constitutes of the two electrons and the negatively charged pion would be, (apparent spin matrix as the spin of each combination of particles correspond to the bosonic superfluidity).

$$
\emptyset=\left(\begin{array}{cc}
0 & 0 \\
1 & -1 \\
2 & -2
\end{array}\right)
$$

And as we know the free Schrödinger equation for this particle is-

$$
\left(\frac{-\hbar^{2} \nabla^{2}}{2 m}+V(r)\right) \psi=E \psi
$$

Since $\mathrm{E}=\mathrm{E}_{\mathrm{f}}+\Delta$,

$$
\left(\frac{-\hbar^{2} \nabla^{2}}{2 m}+V(r)\right) \psi=\left(E_{f}+\Delta\right) \psi
$$

We know that the spin is not a constant so,

$$
\int_{\emptyset=-2}^{\emptyset=2}\left(\frac{-\hbar^{2} \nabla^{2}}{2 m}+V(r)\right) \psi=\left(E_{f}+\Delta\right) \psi
$$


Now, keeping all this in mind let us calculate the critical temperature of the example.

$$
T_{c}=1.13 \times \frac{\hbar \omega}{k_{B}} \times \exp \left(\frac{-1}{N_{0} V_{0}}\right)
$$

Since $\exp \left(\frac{-1}{N_{0} V_{0}}\right)=1$,

$$
\begin{gathered}
T_{c}=1.13 \times \frac{\hbar \omega}{k_{B}} \\
\omega=\frac{m v^{2}}{2 \hbar}
\end{gathered}
$$

Speed of sound in this material is $3200 \mathrm{~ms}^{-1}$ [2].

$$
\begin{gathered}
\omega=\frac{139 \times 1.79 \times 10^{-30} \times 3200^{2}}{2 \times 1.05457 \times 10^{-34}} \\
\omega=1.20 \times 10^{13} \quad 3.39
\end{gathered}
$$

Substituting,

$$
\begin{gathered}
T_{c}=1.13 \times \frac{\hbar \omega}{k_{B}} \quad 3.40 \\
T_{c}=1.13 \times \frac{1.05457 \times 10^{-34} \times 1.2 \times 10^{13}}{1.38 \times 10^{-23}} \\
T_{c}=98 \mathrm{~K} \pm 6 \quad 3.42
\end{gathered}
$$

This matches the experimentally found critical temperature of this superconductor [1]. Hence, there is a good probability of this theory being correct! The main reason this temperature comes so is because the exponential component in the bound state energy for cooper pairs has a certain value, however since the mass of a meson is so much greater than the electron the value becomes 1 and thus it increases the critical temperature for such superconductors which cannot be explained using the BCS theory. 


\section{Potential Well Understanding}

Now, in the final section we will be seeing the quantum potential well representation of the mesonic high temperature superconductive state. The potential well in consideration will have a depth equal to the total internal energy of the pion and will have its boundaries, i.e., L, as the spin states. Thus, it will look something like this,

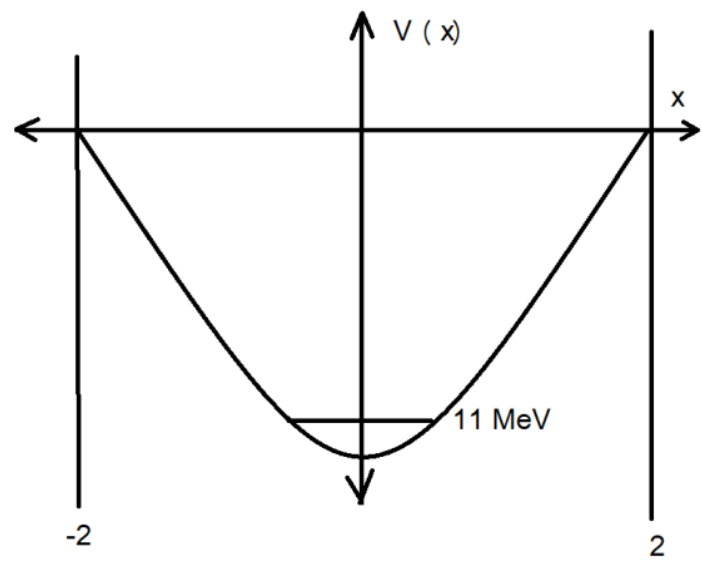

Fig. 4.1

Here the last energy level is the bound state energy at which super conduction occurs and the limits are the minimum and maximum spin states. Now we will be calculating the ground state of this potential well to find out the energy difference to be able to see if it fits in other data.

If $\mathrm{U}_{0}$ is the ground state energy we have,

$$
k \tan \frac{k L}{2}=\sqrt{\beta^{2}-k^{2}}
$$

Where,

$$
E=\frac{\hbar^{2} k^{2}}{2 m}
$$

And,

Solving for k,

$$
\beta=\sqrt{\frac{2 m U_{0}}{\hbar^{2}}}
$$

$$
E=\frac{\hbar^{2} \mathrm{k}^{2}}{2 m} \quad 4.4
$$




$$
\begin{gathered}
k=\frac{\sqrt{E 2 m}}{\hbar} \quad 4.5 \\
k=\frac{\sqrt{139 \times 1.6 \times 10^{-13} \times 2 \times 139 \times 1.79 \times 10^{-30}}}{1.05457 \times 10^{-34}} \quad 4.6 \\
k=9.9756 \times 1.2 \times 10^{6} \quad 4.7 \\
k=11970720 \quad 4.8
\end{gathered}
$$

So,

$$
k \tan \frac{k L}{2}=\sqrt{\beta^{2}-k^{2}} \quad 4.9
$$

Substituting,

$$
k \tan \frac{k L}{2}=\sqrt{\frac{2 m U_{0}}{\hbar^{2}}-k^{2}} \quad 4.10
$$

Since $\mathrm{L}=4$

$$
\begin{gathered}
k \tan 2 k=\sqrt{\frac{2 m U_{0}-k^{2} \hbar^{2}}{\hbar^{2}}} \\
(k \tan 2 k)^{2}+k^{2} \hbar^{2}=2 m U_{0} \\
U_{0}=\frac{(k \tan 2 k)^{2}+k^{2} \hbar^{2}}{2 m} \\
U_{0}=51.56 \mathrm{GeV} \quad 4.14
\end{gathered}
$$

Therefore, the energy difference will be,

$$
\begin{gathered}
\Delta E=E_{f}-E_{i} \quad 4.15 \\
\Delta E=51560-11=51.549 \mathrm{GeV} \quad 4.16
\end{gathered}
$$

Therefore, we can say that the energy of an emitted pion needs to be reduced to $11 \mathrm{MeV}$ for superconductive state. 


\section{Conclusion.}

Some of the elements derived with respect to this theory, having the probability of occurring in substances that can be classified as high temperature superconductors are found abundantly on earth and some are even occurring in experimentally found high temperature superconductors. Many different lattices can be described using the format given before with respect to the examples mentioned previously. The potential described before can also contribute to other forms of superconductivity, which may will increase the critical temperature even more; these are yet to be found though.

\section{References.}

\section{Citations:}

1. King, F., Finding the critical temperature of a YBCO superconductor using a voltage probe. Physics Department, The College of Wooster, 2008: p. 1-4.

2. Takanohashi, T. "Ultrasonic study of YBa2Cu3O7-x superconductors." Le Journal de Physique IV 6.C8 C8-473 (1996):.

3. Cohen, B. L. (1971). Concepts of nuclear physics.

4. Galitski, Victor, Boris Karnakov, and Vladimir Kogan. Exploring Quantum Mechanics: A Collection of 700+ Solved Problems for Students, Lecturers, and Researchers. OUP Oxford, 2013. Galitski, Victor, Boris Karnakov, and Vladimir Kogan. Exploring Quantum Mechanics: A Collection of 700+ Solved Problems for Students, Lecturers, and Researchers. OUP Oxford, 2013.

5. Timm, Carsten. "Theory of superconductivity." Institute of theoretical Physics Dresden (2012).

6. Lakhno, Viktor Dmitrievich. "Translation invariant bipolarons and charge density waves in high-temperature superconductors." Preprints of the Keldysh Institute of Applied Mathematics (2020): 57-13.

\section{Other references:}

- Exploring Quantum Physics, offered by Coursera.org, given by The University of Maryland, with lecturers - Dr. Victor Galitski \& Charles W. Clark.

- Lattice of YBaCuO from www.wikipedia.org.

- https://www.google.com/url?sa=i\&url=http\%3A\%2F\%2Fhydrogen.physik.uniwuppertal.de\%2Fhyperphysics \%2Fhyperphysics \%2Fhbase \%2Fquantum\%2Fpfbox.html \&psig=AOvVaw093cw7bBxa7rKoXJD9D1CZ\&ust=1590073575559000\&source=image $\underline{\text { s\&cd=vfe\&ved=0CAMQjB1qFwoTCIjRz-nbwukCFQAAAAAdAAAAABAI }}$ 